\title{
A program for comprehension monitoring of text using HyperCard for the Macintosh
}

\author{
STEVE NASON and KAREN ZABRUCKY \\ Georgia State University, Atlanta, Georgia
}

\begin{abstract}
We describe a program using HyperCard for the Macintosh that allows the collection of several different types of text processing data in a realistic, natural, and unobtrusive experimental setting. The program allows a sentence-by-sentence presentation of text and allows unconstrained movement forward and backward through the text. Subjects can, at any point during reading, look back at individual sentences presented previously, or review all previously presented sentences at once. All sentence readings and rereadings are timed, and the sequence of readings, rereadings, and reviews is recorded. Following passage reading, questions are asked to examine comprehension and memory of the passage. The program is well suited for the examination of comprehension evaluation and regulation in readers, as well as for the examination of other comprehension and memory processes.
\end{abstract}

Over the last several years psychologists have become increasingly interested in studying comprehension and memory of complex discourse information. Early investigations (e.g., Bransford \& Johnson, 1972; Dooling \& Lachman, 1971) included simple analyses of the factors contributing to text comprehensibility and memorability, often involving comprehension ratings and idea units recalled as the primary dependent variables. From such preliminary studies, investigations of text processing evolved into a systematic examination of the cognitive processes involved in comprehending, integrating, and remembering textual information. This evolution was dramatically helped by the development and availability of inexpensive computers that allowed on-line examinations of comprehension processes. With the help of on-line systems, research began in earnest on many processes, for example, on the effects of text coherence on comprehension and memory (see Cirilo, 1981; Lesgold, Roth, \& Curtis, 1979; Zabrucky, 1986). The investigation of many processes required relatively simple on-line programs, for example, those that allowed measurement of individual sentence reading times. However, examinations of other reading processes have been hindered until now, because of the unavailability of on-line systems that did not interfere with reading as it occurs naturally and that also allowed measurement of a variety of reading activities.

The program described here used the HyperCard for the Macintosh, a new software "construction" program from Apple Computer that allows unobtrusive measurement of relatively complex reading processes. The program has been designed to examine comprehension monitoring, a multidimensional process involving both comprehension evaluation and comprehension regulation.

Requests for reprints should be sent to Karen Zabrucky, Department of Educational Foundations, Georgia State University, University Plaza, Atlanta, GA 30303.
Evaluation has most commonly been measured using the "error detection" paradigm: a problem of some sort (e.g., a passage inconsistency) is inserted into an otherwise intact text. Detection of the problem is taken as evidence that a reader can evaluate his or her own understanding. Detection has most often been measured on the basis of verbal reports following reading, such as responses to probe questions (e.g., "Did the story make sense?"), or on the basis of ratings of passage comprehensibility (Garner, 1980, 1981; Markman, 1979; Winograd \& Johnston, 1982). In a recent paper, Zabrucky and Ratner (1986) discuss the benefits of using on-line measures such as reading times in conjunction with verbal report measures obtained following reading to examine evaluation. They also argue that on-line measures allow a muchneeded examination of regulation strategies used by readers to resolve comprehension failures, such as rereading text information. These strategies have been virtually ignored because of the unavailability of on-line systems that can measure such behaviors unobtrusively.

An important requirement in using an on-line system to examine regulation is that it allows subjects to read naturally, without having to manipulate a computer keyboard in a complex manner. If a process such as rereading requires complex actions (e.g., several movements on a computer) in the laboratory, then subjects may simply not engage in the process. Indeed, failures to find evidence of rereading in previous research (see, e.g., August, Flavell, \& Clift, 1984) may have occurred partly because on-line systems required too much effort on the part of subjects.

Because of the limitations of available computer systems, there have been some recent attempts to investigate regulation processes in a natural and unobtrusive manner without the use of a computer. For example, Zabrucky and Ratner (1986) developed a videotaping procedure which allowed children to read ahead or look back in pas- 
sages presented one sentence at a time very easily and thus had an advantage over previously used on-line systems. Unfortunately, however, Zabrucky and Ratner's procedure is quite time consuming. It requires extensive coding of videotapes, as well as trained judges to score the videotapes for both reading times and look-backs. In addition, the procedure allows an examination of only one type of look-back, namely look-backs to individual sentences presented previously in the text.

The on-line system described here allows an examination of individual sentence reading times, the number of look-backs to individual sentences, the pattern of lookbacks employed, and procedures such as the review of all previously presented material. The system does not require extensive coding and scoring of information, or the use of trained judges. The data can be viewed or printed immediately after the completion of an experimental session. Passage sentences can be presented individually in a self-paced manner so that individual sentence reading times can be obtained. The program allows the subject to look back at one or more sentences at any time. The reading times for look-backs are obtained, as is the pattern of the subject's look-back movements. Following passage reading, questions are asked to examine the subject's comprehension and recognition of information presented in the text. Question content can easily be altered so that one may study other comprehension or memory processes; the data from questions can be combined with recall protocols so that one may obtain more comprehensive information regarding the subject's memory of text information.

\section{Equipment}

HyperCard currently operates only on a Mac Plus, Mac SE, or Mac II; it requires a minimum of one megabyte of RAM and 128K ROMS. Although the program will operate with two $800 \mathrm{~K}$ disk drives, a significant improvement is achieved with a hard disk. Printers with appropriate driver software, such as the Imagewriter I or II, can be used to obtain hard copy of the data or individual screens. The standard Macintosh mouse, as well as a trackball, an optical mouse or a graphics tablet, can be used as the pointing device.

\section{Software}

Hypercard is based in part on the concept of hypertext (Nelson, 1965). Hypertext refers to the interconnection of text and graphical material in complex links not readily represented in a single medium. The concept is applicable to many complex cognitive processes such as reading or writing. For example, during the course of reading a passage, an individual may make notes about some idea for later use, look up definitions in a dictionary, or reread sentences to aid comprehension. Hypertext allows individuals to use such different types of information whenever they wish, as often as they wish, and then continue with the original task.

\section{Comprehension Monitoring Program}

The program described here allows a sentence-bysentence presentation of text and an unconstrained movement forward and backward through the text. The subject can, at any point during reading, look back at individual sentences presented previously, or review all earlier sentences at once. All sentence readings and rereadings are timed, and the sequence of readings, rereadings, and reviews is recorded.

The screen looks like a notebook: pages appear to be turned forward or backward by clicking the mouse on arrows pointing to the right or left, respectively. The arrows appear at the upper right-hand corner of each screen page. The first two screen pages consist of instructions that provide a description of the reading activities available to the subject. These activities include moving to the next page, moving to the previous page, reviewing all previously read sentences at once all sentences read previously, or reexamining the instructions. The activities can be initiated at any time during the reading of a passage, simply by clicking the mouse on the right or left arrows on the upper right-hand corner of the screen, or on "Review" or "Instructions" buttons at the bottom of each screen page. After using the "Instructions" or the "Review" button, the subject simply clicks on a "Go Back" button at the bottom of the page to return to the original location in the text. The subject is given an opportunity to practice the mouse commands and then on an additional page receives instructions to begin reading the passage. The subject is instructed to read each sentence in the passage carefully and to continue reading sentences until finished.

After the instructions, a passage is presented, one sentence per screen (see Figure 1). ${ }^{1}$ Once the first sentence of a passage has been presented, the subject can, at any time, look back at any or all of the sentences, or view the program instructions. The amount of time spent at each screen during the reading of a passage is recorded in six-

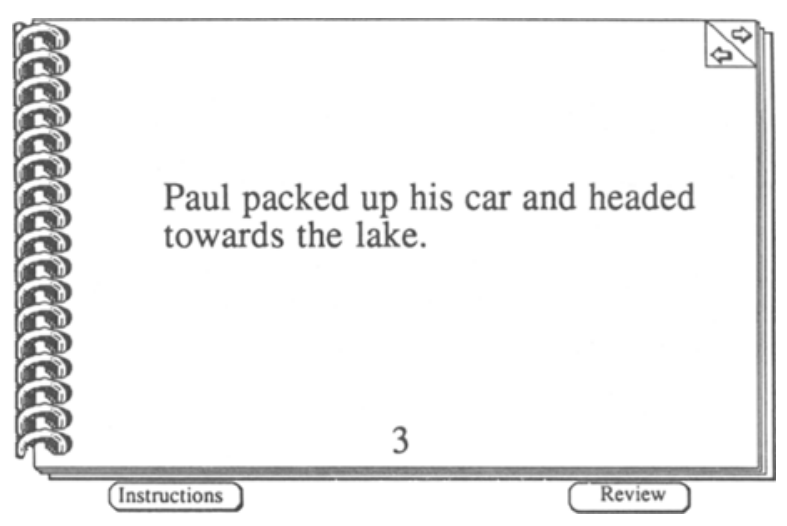

Figure 1. Screen page showing a presented sentence. 


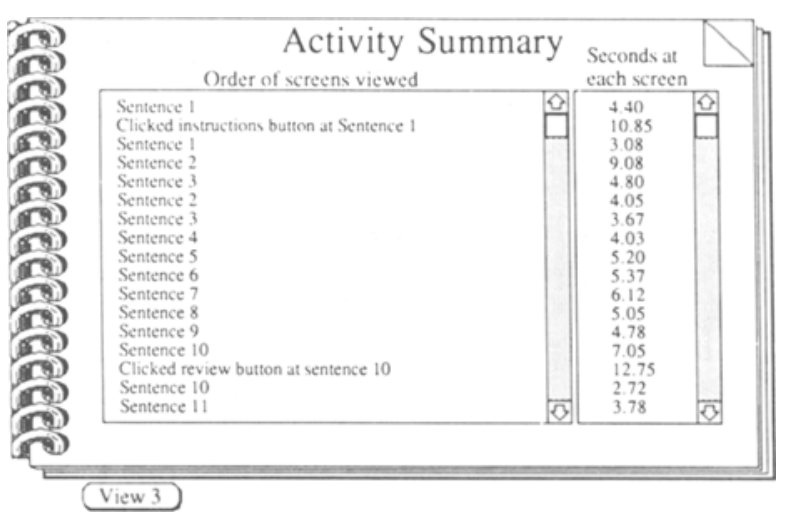

Figure 2. Screen page showing an example Activity Summary.

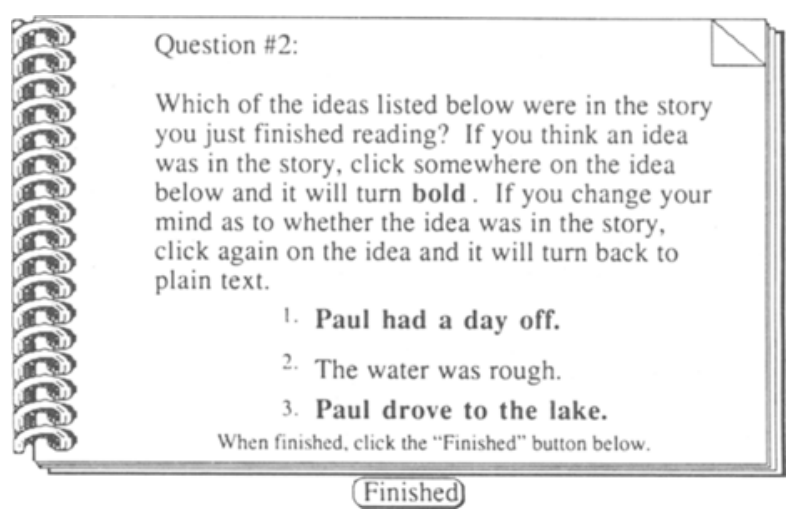

Figure 3. Screen page showing selections of ideas present in the passage.

tieths of a second. The sequence of movements from one screen to another is recorded, providing an Activity Summary of the subject's actions.

Figure 2 shows an example of an Activity Summary for a subject. The column labeled "Order of Screens Viewed" indicates the order in which sentences were read and when the subject clicked on the "Instructions" or the "Review" button. Thus, for example, the example Activity Summary indicates that the subject read Sentence 1, reread the instructions (going back, or course, to Sentence 1 after this action), read Sentence 2, read Sentence 3, reread Sentences 2 and 3, read Sentences 4 through 10 , reviewed all previously read sentences (going back to Sentence 10 after this action), and then read Sentence 11. The column marked "Seconds at Each Screen" shows the amount of time spent for each activity.

Following passage reading, questions are asked to examine a subject's comprehension and memory of the passage. In the present program, one question is asked in order to assess comprehension evaluation ability, and others are asked in order to assess recognition memory of information from the passage. The order and content of these questions can of course be changed to suit the investigator's needs.
On the first screen page following the reading of the passage, a probe question frequently used in comprehension evaluation research (i.e.,"Did the story make sense?") is asked. The question is answered by clicking either a "Yes" or a "No" button that appears below the question; this records the selection for later examination. On the next screen, the subject is shown a list of idea units and is asked to click on the idea units presented in the passage. Clicking on any individual idea unit highlights it and records the selection for later examination. The subject may change his or her mind by clicking again on the idea unit. This action dehighlights the idea unit and removes it from the recorded responses. A screen page that illustrates an idea unit presented in the passage is shown in Figure 3. The recorded selections are provided in an "Ideas" Summary, as shown in Figure 4.

On the next two screens, questions are asked in order to assess recognition memory of specific story content. In the present program, the questions assess recognition memory for inconsistent sentences in the passage (i.e., "Did the story say that Paul was sad that he forgot to pack his fishing pole?" and "Did the story say that Paul caught a big fish with his fishing pole?"'). Each question is answered by clicking either a "Yes" or a "No" button appearing below the question. The recorded selections for

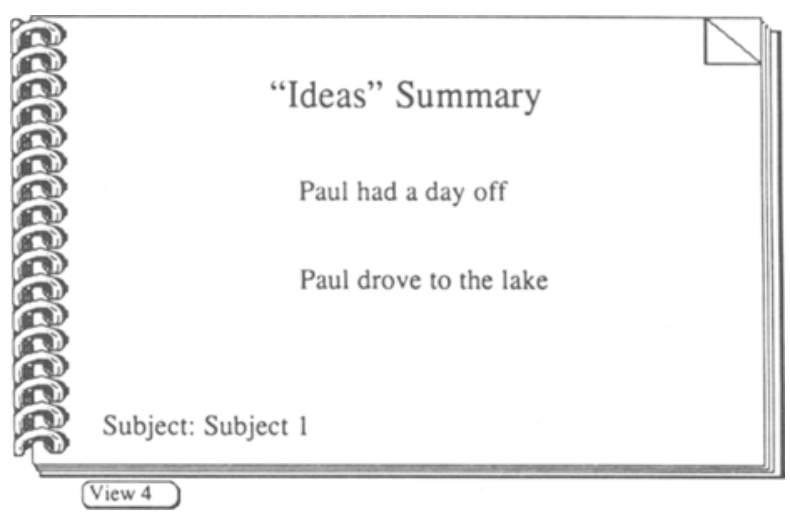

Figure 4. Screen page showing an example "Ideas" Summary.

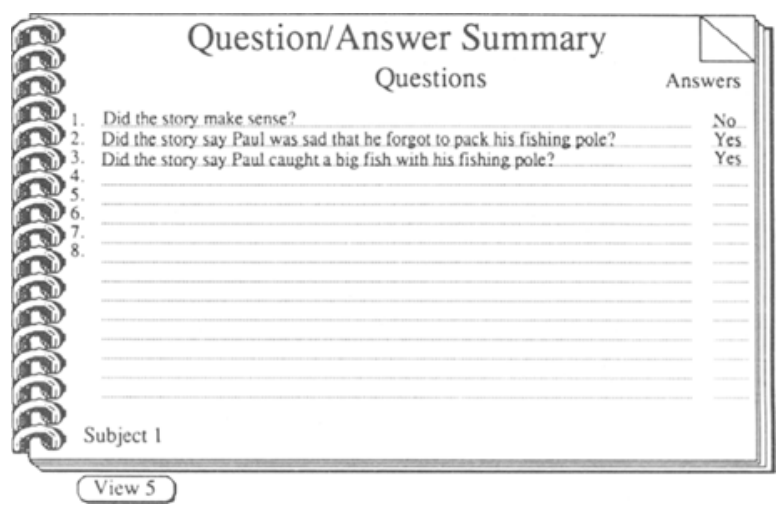

Figure 5. Screen page showing an example Question/Answer Summary. 
these recognition memory questions as well as the probe question are provided in a Question/Answer Summary, as shown in Figure 5.

After the subject has answered the questions, the investigator can view or print the subject's data, which includes the Activity Summary, the "Ideas" Summary, and the Question/Answer Summary (Figures 2, 4, and 5). A list of all the sentences presented to the subject can also be viewed and printed.

The data allow an investigator to analyze several dependent measures important in comprehension monitoring research, such as reading times of individual passage sentences, overall passage reading time, number of lookbacks, time spent at each look-back, responses to probe questions, and recognition of idea units or of specific information in passage sentences. Since reading times of individual sentences can be obtained as well as question data, the program is useful for investigating a variety of comprehension and memory processes.

\section{Other Advantages of the System}

There are a number of other advantages of the present on-line system using HyperCard on a Macintosh computer. First, the system is extremely portable, allowing field research to be more feasible. Second, the screen resolution is excellent, resulting in highly readable text. Third, text information can be presented in the entire range of font sizes and styles available on the Macintosh. This important advantage allows investigators to use special fonts for special populations-for example, large fonts for young children or elderly adults. Fourth, screen pages can be printed at any time during the program, allowing hard copies of any stimuli or data information desired. Finally, although not used in the present program, HyperCard allows presentation of graphic material (from line drawings to digitized images) and digitized sound and has extensive graphic capabilities allowing the ability to degrade or enhance visually presented information. For these reasons, on-line systems using HyperCard allow a variety of experimental stimuli to be presented.

\section{Availability}

The stack and associated documentation may be obtained on disk for $\$ 10.00$ by writing Karen Zabrucky,
Department of Educational Foundations, Georgia State University, University Plaza, Atlanta, Georgia 30303.

\section{REFERENCES}

August, D. L., Flavell, J. H., Clift, R. (1984). Comparison of comprehension monitoring of skilled and less skilled readers. Reading Research Quarterly, 20, 39-53.

Bransford, J. D., Johnson, M. K. (1972). Contextual prerequisites for understanding: Some investigations of comprehension and recall. Journal of Verbal Learning \& Verbal Behavior, 11, 717-726.

CiRILo, R. K. (1981). Referential coherence and text structure in story comprehension. Journal of Verbal Learning \& Verbal Behavior, 20, 358-367.

Dooling, D. J., \& Lachman, R. (1971). Effects of comprehension on retention of prose. Journal of Experimental Psychology, 88, 216-222.

GARNER, R. (1980). Monitoring of understanding: An investigation of good and poor readers' awareness of induced miscomprehension of text. Journal of Reading Behavior, 12, 55-63.

GaRNER, R. (1981). Monitoring of passage inconsistency among poor comprehenders: A preliminary test of the "piecemeal processing" explanation. Journal of Educational Research, 74, 159-162.

LesGold, A. M., Roth, S. R., \& CuRTIS, M. E. (1979). Foregrounding effects in discourse comprehension. Joumal of Verbal Learning \& Verbal Behavior, 18, 291-308.

Markman, E. M. (1979). Realizing that you don't understand: Elementary school children's awareness of inconsistencies. Child Development, 50, 643-655.

Nelson, T. (1965). A file structure for the complex, the changing, and the indeterminate. Proceedings of the 20th Annual National Conference of the Association for Computing Machinery, 6, 85-99.

Winograd, P., \& Johnston, P. (1982). Comprehension monitoring and the error detection paradigm. Journal of Reading Behavior, 14, 61-76.

ZABRUCKY, K. (1986). The role of factual coherence in discourse comprehension. Discourse Processes, 9, 197-220.

ZABRUCKY, K., \& RATNER, H. H. (1986). Children's comprehension monitoring and recall of inconsistent stories. Child Development, 57, 1401-1418.

\section{NOTE}

1. All sentences used in the present paper are from Zabrucky and Ratner (1986).

(Manuscript received February 24, 1988; revision accepted for publication June 4,1988 .) 